\title{
Statement of ESMINT and ESNR regarding recent trials evaluating the endovascular treatment at the acute stage of ischemic stroke
}

\author{
L. Pierot • M. Söderman • M. Bendszus • P. White • \\ M. Muto $\cdot$ F. Turjman $\cdot$ S. Mangiafico $\cdot$ J. Gralla $\cdot$ \\ J. Fiehler • I. Szikora $\cdot$ C. Cognard
}

Received: 9 July 2013 / Accepted: 17 July 2013 /Published online: 20 August 2013

(C) Springer-Verlag Berlin Heidelberg 2013

\section{Background}

Three recent, simultaneous publications report the neutral results of three randomized studies (SYNTHESIS Expansion, Interventional Management of Stroke (IMS) III, and Mechanical Retrieval and Recanalization of Stroke Clots Using Embolectomy (MR RESCUE)) comparing IV thrombolysis therapy or standard care (MR RESCUE) to the endovascular treatment (EVT) of acute ischemic stroke (AIS) [1-3]. The results of these three trials might lead to the erroneous conclusion that endovascular treatment has no or little place in the management of AIS. However, careful analysis of the IMS III, SYNTHESIS, and MR RESCUE

L. Pierot

Neuroradiology, Hôpital Maison Blanche, Université Reims

Champagne-Ardenne, Reims, France

\section{Söderman}

Neuroradiology, Karolinska Hospital, Stockholm, Sweden

\section{Bendszus}

Neuroradiology, UniversitätsKlinikum Heidelberg,

Heidelberg, Germany

P. White

Institute for Ageing and Health, Newcastle University Stroke

Research Group, Newcastle upon Tyne, UK

M. Muto

Neuroradiology, Cardarelli Hospital, Naples, Italy

F. Turjman

Neuroradiology, Hôpitaux de Lyon, Lyon, France

S. Mangiafico

Neuroradiology, AOU Careggi, Florence, Italy studies is mandatory prior to assessment of the value of EVT of AIS. Also, the limits of the current and future trials should be outlined.

The European Society of Minimally Invasive Neurological Therapy (ESMINT) and European Society of Neuroradiology (ESNR) represent the interventional neuroradiology community in Europe and have engaged in the definition of rules regarding trials evaluating the EVT of AIS [4, 5]. It is clearly their role to provide a precise analysis of recent and future trials.

In the last 20 years, treatment of AIS has evolved to include neuroprotection, IV thrombolysis (IVT), and endovascular treatment. Despite numerous studies of a very large number

J. Gralla

Neuroradiology, Inselspital, University of Bern,

Bern, Switzerland

J. Fiehler

Neuroradiology, University Medical Center

Hambourg-Eppendorf, Hamburg, Germany

I. Szikora

Neuroradiology, National Institute of Neurosciences,

Budapest, Hungary

C. Cognard

Neuroradiology, CHU Toulouse, Toulouse, France

L. Pierot $(\square)$

Service de Neuroradiologie, Hopital Maison Blanche, 45, rue Cognacq Jay, 51092 Reims cedex, France e-mail: lpierot@gmail.com 
of drugs, none of them have demonstrated efficacy in the protection of ischemic brain [6]. Several randomized studies of IV thrombolysis (IVT) were negative before it was first reported that IVT therapy administered within the first $3 \mathrm{~h}$ after stroke onset had positive clinical effects [7-11]. IVT therapy was approved by the FDA in 1995. This result was subsequently confirmed by other studies, leading to an extension of the therapeutic window to $4.5 \mathrm{~h}$ [12-15].

The EVT of AIS has rapidly evolved from intra-arterial (IA) chemical thrombolysis to mechanical thrombectomy. IA chemical thrombolysis was evaluated in several randomized trials showing its efficacy and safety (versus placebo and not versus IV recombinant tissue plasminogen activator (IV rtPA) as it was not authorized at the time of these trials) [16-20]. The rationale for IA chemical thrombolysis was to infuse the thrombolytic drug as close as possible to the clot (proximal, inside, or distal to the clot), in order to increase the local concentration of the drug, while simultaneously limiting its systemic concentration. The next step in EVT of AIS was mechanical thrombectomy. Originally, this was performed with no specific tools (injection of saline within the clot, disruption of the clot with a microguidewire, "angioplasty" of the clot with remodeling balloons). Subsequently, dedicated first-generation devices, such as the MERCI and Penumbra devices, were developed to catch or aspirate the clot [21-23]. They were not compared to IV rTPA in randomized controlled trials (RCTs). Second-generation devices (e.g., "stentrievers" such as Solitaire ${ }^{\mathrm{TM}}$ ) were developed to promptly restore blood flow through retrieval of the clot and were initially evaluated in a small, single center series [24-26].

There has been no RCT that compared EVT to IV rTPA for the management of AIS. IMS III, SYNTHESIS, and MR RESCUE are the first RCTs comparing endovascular treatment to IV rTPA or standard care for the management of AIS. The teams who have conducted these trials have to be congratulated, as this has required considerable effort.

\section{IMS III}

IMS III is an international, phase 3, randomized, open-label clinical trial with a blinded outcome, comparing a combined approach of IV rTPA followed by endovascular treatment with standard IV rTPA treatment. The goal was to include 900 patients (300 in IV rTPA group and 600 in EVT group) in order to detect a $10 \%$ absolute risk reduction for disability and death. IV rTPA (standard dose of IV rTPA, $0.9 \mathrm{mg}$ per kilogram) was started within $3 \mathrm{~h}$ after symptom onset in both arms. Patients in the EVT group underwent angiography as soon as possible, and those with a treatable occlusion received endovascular treatment with an approach chosen by the site interventionalist (i.e., thrombectomy with the MERCI retriever (Concentric Medical), Penumbra system (Penumbra), or endovascular delivery of rTPA by means of the MicroSonic SV infusion system (EKOS) or a standard microcatheter). The Solitaire ${ }^{\mathrm{TM}} \mathrm{FR}$ stent-retriever was introduced in the late stage of the trial after approval by the FDA.

From August 2006 to April 2012, 656 participants in 58 centers underwent randomization (434 participants to endovascular therapy and 222 to IV rTPA alone). The trial was stopped early due to futility, according to pre-specified rules. The proportion of patients with good [Rankin] outcome was slightly, but not significantly, higher in the endovascular group ( $40.8 \%$ in the endovascular therapy arm and $38.7 \%$ in the IV rTPA arm). There was also no significant difference in mortality at 90 days between the two groups $(19.1 \%$ in the endovascular therapy group and $21.6 \%$ in the IV rtPA group), as well as in the rate of symptomatic intracerebral hemorrhage within $30 \mathrm{~h}$ after initiation of rTPA $(6.2 \%$ in the endovascular therapy group and $5.9 \%$ in the rTPA group).

IMS III had several important drawbacks that considerably limits its value:

(1) The inclusion period was very long ( 68 months $=5$ years 8 months) in a very large number of centers (58). The number of patients included per year per center was around two patients (1.3 in the EVT group and 0.7 in the IV rtPA group), but it is not taking into account the fact that all centers were not simultaneously activated. This very low number has several consequences:

- IMS III represents a very strong selection of patients as it is difficult to imagine that only two patients per year were referred to the participating centers for treatment of AIS. Enrollment of patients in IMS III was probably not consecutive with some patients not included in the trial for compassionate use of endovascular treatment. Unfortunately, no information is given regarding the number of patients directly treated with EVT during the study period in the participating centers.

- As the number of patients/center is very low, it is clear that it is difficult for interventionalists to develop expertise in the EVT. In addition, it is probable that lack of routines for EVT delayed the management considerably.

- During the long inclusion period, imaging and endovascular treatment modalities underwent a tremendous evolution, and utilization of the new technology was only partially implemented in the IMS III protocol (see below).

(2) EVT may be effective only in patients having major arterial occlusion at the level of the circle of Willis (internal carotid artery, middle cerebral artery, and basilar artery). At the beginning of the trial, pre-therapeutic imaging was mostly plain $\mathrm{CT}$ and no $\mathrm{CT}$ angiogram (CTA) was performed. CTA was introduced in the mid- 
course of the trial and only 306/656 participants (46.6\%) had preoperative CTA. CTA was not used for selection of patients and National Institutes of Health Stroke Scale (NIHSS) score was used to identify patients with a score of 10 or more, who had greater than $80 \%$ likelihood of major arterial occlusion. This methodology is clearly very poor.

Of the patients with baseline CTA, 24/306 patients (7.8\%) had no arterial occlusion, with 20 of these patients randomized to the endovascular group and 4 to the IV rTPA group. Additionally, 89/423 patients $(21.0 \%)$ who had received angiograms were allocated to the endovascular group and ultimately did not have endovascular treatment. It means that due to the protocol design, some patients were exposed to the risks of angiography without any benefits in terms of treatment. According to IMS III protocol (changed in 2011), these patients received an IV rTPA dose less than the standard dose, which could contribute to the negative results of the trial. Clearly, the inclusion of patients without a major arterial occlusion in a randomized trial dealing with endovascular recanalization is a major weakness of the IMS III trial.

Interestingly, in patients for whom CTAs were obtained, the rate of partial or complete recanalization was different in the endovascular therapy and IV rTPA groups (respectively, 81 and $35 \%$ for an occlusion in the internal carotid artery, 86 and $68 \%$ for an M1 occlusion, and 88 and $77 \%$ for an M2 occlusion). In terms of recanalization, mechanical thrombectomy performed better than IV rTPA alone for all locations, and as demonstrated in IMS III, the proportion of patients with good clinical outcome increased with greater reperfusion. Unfortunately the 90-day clinical outcome in patients with CTA-confirmed occlusion at baseline is not analyzed and we don't know if EVT performed better than in rTPA alone in this sub-group of patients.

(3) An important limitation of the IMS III study is that the modalities of endovascular treatment were very heterogeneous and some became obsolete during the course of the trial: $164 / 434$ patients $(37.8 \%)$ were treated by IA rTPA administration through a standard or EKOS microcatheter, 149/434 (34.3\%) by mechanical thrombectomy with first-generation devices (MERCI or Penumbra), and only 5/434 (1.2\%) by mechanical thrombectomy with a second-generation device, such as the Solitaire ${ }^{\mathrm{TM}}$ FR device. As the SWIFT and TREVO2 trial have clearly demonstrated, the secondgeneration devices are more efficacious than firstgeneration devices in terms of both recanalization and clinical outcome (see below) [27, 28].

(4) Finally, time from stroke onset to initiation of IV rTPA (mean) was $121 \mathrm{~min}$ in the IV rTPA group alone and
$122 \mathrm{~min}$ in the endovascular therapy group. In the endovascular therapy group, time from onset to groin puncture and to IA therapy were (means) $208 \mathrm{mn}$ and 249 min respectively. The endovascular treatment started at $86 \mathrm{mn}$ and terminated at $127 \mathrm{mn}$ after onset of IV rTPA administration. The delay before EVT is an important factor that certainly could affect outcome. The reduction of these delays is mandatory in future trials.

\section{Synthesis}

SYNTHESIS is an Italian, randomized, multicenter clinical trial with a blinded endpoint, comparing standard IV rTPA treatment (initiated within $4.5 \mathrm{~h}$ after symptom onset) to endovascular treatment (within $6 \mathrm{~h}$ after symptom onset). All patients underwent randomization within $4.5 \mathrm{~h}$ of symptom onset. Patients who were assigned to the endovascular treatment group did not receive IV rTPA. Several endovascular treatments were authorized in the trial including IA administration of rTPA (maximum dose of $0.9 \mathrm{mg}$ per kilogram of body weight), or mechanical thrombectomy, including the use of a microguidewire to disintegrate the clot or of systems to capture, crush or aspirate the clot. Again, the demonstration of vessel occlusion was not a precondition for inclusion in this trial.

From February 2008 to April 2012 (51 months), 362 patients underwent randomization (181 to endovascular treatment and 181 to IV rTPA) in 24 centers. In the endovascular group, 15 patients did not receive treatment due to clinical improvement, lack of evidence of occlusion, or other causes. 165 patients received endovascular treatment (without an equipment breakdown requiring interruption). Endovascular treatment with IA rTPA and fragmentation of the thrombus with a guidewire was performed in 109/165 patients (66.1\%). A device was used in 56/165 patients (33.9\%). These devices included first-generation devices (MERCI, Concentric and Penumbra, Penumbra) in 14/165 patients (8.5\%) and secondgeneration devices (Solitaire ${ }^{\mathrm{TM}}$, Covidien/ev3 or Trevo, Concentric) in 23/165 patients (13.9\%). The primary endpoint (disability-free survival at 90 days, modified Rankin Scale (mRS) 0 or 1) was similar in both groups $(30.4 \%$ in the endovascular group and $34.8 \%$ in the IV rTPA group). Symptomatic intracranial hemorrhage within 7 days occurred in $6 \%$ of patients in both groups. Additionally, death at 90 days was not significantly different between the two groups (14.4\% in the endovascular group and $9.9 \%$ in the IV rTPA group).

The inclusion period was slightly shorter for Synthesis than for IMS III, but the same limitations are encountered in SYNTHESIS:

(1) Even if slightly higher, the number of patients included per year in the centers was very low (3.5 patients/year/ 
center) with the same consequences than in IMS III (see above).

(2) As in IMS III, advanced CT or MR modalities were not used to visualize vessel occlusion and the number of patients without arterial occlusion is not reported. See above.

(3) Also, endovascular treatment modalities were quite heterogeneous and not infrequently obsolete (IA rTPA combined with guidewire disintegration of the clot in $66.1 \%$, EVT with first-generation devices in $8.5 \%$ and secondgeneration devices in only $13.9 \%$ ).

Other important limitations that were encountered in SYNTHESIS (but not IMS III):

(1) Patients with an NIHSS score as low as 2 were included. They have a very high probability of having a good recovery at 3 months regardless of treatment given.

(2) IV rTPA was not given in the endovascular group. Synthesis was, in fact, comparing IV rTPA treatment to isolated endovascular treatment and not to combined IV rTPA treatment and mechanical thrombectomy. As a consequence, the endovascular group received treatment $1 \mathrm{~h}$ later than the IV rTPA group, which may have impact on the relative equivalence of endovascular treatment and IV rTPA treatment.

(3) The recanalization rate is not reported and analyzed.

\section{MR RESCUE}

The MR RESCUE trial was a small phase $2 b$, randomized, controlled, open-label, multicenter trial conducted at 22 sites in North America. Patients between the ages of 18 and 85 years with NIHSS scores of 6 to 29 who had a largevessel, anterior circulation ischemic stroke were randomly assigned within $8 \mathrm{~h}$ after the onset of symptoms to undergo either mechanical embolectomy (MERCI retriever or Penumbra System) or to undergo undefined standard medical care. All patients underwent pretreatment multimodal CT or MRI of the brain, which permitted stratification according to the presence of a favorable penumbral pattern versus a nonpenumbral pattern. Among 118 eligible patients (recruited over 8 years), 64 were assigned to the embolectomy group (the patients were treated with US-authorized devices, MERCI at the beginning of the trial, then Penumbra) and 54 were assigned to the standard care group (including treatment with IV rTPA). In total, 68/118 patients (57.6\%) were found to have a favorable penumbral pattern following pretreatment imaging. The 118 patients were classified into four groups: embolectomy/penumbral (34 patients); standard care/ penumbral (34); embolectomy/non-penumbral (30); and standard care/non-penumbral (20). Statistical analysis testing to determine whether there was an interaction between treatment assignment and penumbral pattern determined no significance. The number of patients with good outcome at 90 days (mRS 0 to 2) was not significantly different between the groups (embolectomy/penumbral=21\%; standard care/ penumbral $=26 \%$; embolectomy $/$ non-penumbral $=17 \%$; standard care/non-penumbral $=10 \% ; p=0.48)$. Death rates also did not significantly differ (embolectomy/penumbral= $18 \%$; standard care/penumbral $=21 \%$; embolectomy/nonpenumbral $=20 \%$; standard care/non-penumbral $=30 \%$; $p=0.75$ ). Interestingly, reperfusion (assessed on day 7 with the use of perfusion MRI) and partial or complete revascularization (defined as a TICI score of $2 \mathrm{a}$ to 3 ) were not significantly different among groups.

Unlike the IMS III and SYNTHESIS studies, pretreatment evaluation was more precise, with CTA or magnetic resonance angiogram (MRA) used to depict large-vessel proximal anterior circulation occlusion, and multimodal CT or MRI of the brain was used to evaluate penumbral status.

However, the MR RESCUE trial still had several drawbacks:

(1) The number of patients in each group was very small ( $\leq 30$ patients), leading to a high risk for a false negative result (error type II)

(2) As in SYNTHESIS and IMS III, first-generation thrombectomy devices were used.

(3) As in SYNTHESIS, IV rTPA was not given in the endovascular group.

(4) Additionally, it is clear from this trial that the management and use of penumbral patterns was difficult. Despite the development of specific models, the study software processed only $58 \%$ of the cases successfully in real time. Additionally, final pattern assignment changed after core laboratory post-processing in an alarmingly high percentage of cases $(8 \%)$. Unfortunately, it is difficult to know if imaging of penumbra is, at present, a reliable tool for the pretreatment evaluation of AIS patients. Also, the use of two different imaging modalities (CT and MR) to evaluate penumbra may complicate matters further. It should also be noted that the time to enrollment for all patients in this study, regardless of assigned group, was relatively long (from 5.2 to $5.8 \mathrm{~h}$ ).

\section{Discussion}

IMS III, SYNTHESIS, and MR RESCUE are three important trials that illustrate the difficulties in designing studies for techniques that are in rapid evolution. The combined stroke task force of two European societies of neuroradiologists/ neurointerventionists (ESNR-ESMINT) emphasized these issues prior to the publication of the results of the three trials $[4,5]$. The main weaknesses of these trials are: 
- Heterogeneity of the endovascular techniques used with most of them now being obsolete.

- Inappropriate preoperative imaging including absence of CTA or MRA to detect an occlusion of a major arterial trunk (IMS III and SYNTHESIS).

- Inclusion of patients with very low NIHSS (SYNTHESIS).

- Long period of inclusion (all studies).

- Small number of patients per center per year (all studies). This points to lack of expertise and routines,

- No evaluation of the salvageable brain with perfusion CT or MR (IMS III and SYNTHESIS).

IA administration of fibrinolytics, as well as treatment with first-generation thrombectomy devices, has been replaced with stent-based thrombectomy devices. The clinical impact of the use of these novel devices has been impressively illustrated by the recent Solitaire ${ }^{\mathrm{TM}}$ with the intention for thrombectomy (SWIFT) trial which directly compared mechanical thrombectomy with MERCI (first generation) and Solitaire ${ }^{\mathrm{TM}}$ (second generation) devices in a randomized trial. In a global population of 113 patients $\left(\mathrm{MERCI}=53\right.$, Solitaire $\left.{ }^{\mathrm{TM}}=58\right)$, successful recanalization without symptomatic intracranial hemorrhage (primary endpoint) was achieved in $61 \%$ of patients in the Solitaire ${ }^{\mathrm{TM}}$ group and $24 \%$ or patients in the MERCI group $(p<0.0001)$ [27]. Outcomes were significantly better for patients treated with the Solitaire $^{\mathrm{TM}}$ device. Good neurological outcome at 90 days was achieved in $58 \%$ of patients in the Solitaire ${ }^{\mathrm{TM}}$ group and $33 \%$ in the MERCI group $(p=0.0001)$. Mortality at 90 days was $44 \%$ in the MERCI group and $18 \%$ in the Solitaire ${ }^{\mathrm{TM}}$ group $(p=0.0001)$. Similar results were reported for another secondgeneration device, Trevo, in the Trevo 2 trial, confirming that the present generation mechanical thrombectomy devices perform better than their first-generation counterparts [28]. If the superiority of second-generation devices was clearly demonstrated in SWIFT and TREVO 2, the superiority of mechanical thrombectomy with second-generation devices versus IV rTPA is still unproven. Moreover, it should be outlined that the rate of good clinical at 90 days is not different in the endovascular group of IMS III (40.8\%) and in the Solitaire arm of SWIFT (37\%) as well as 90-day mortality rate (19.1\% in the endovascular arm of IMS III and $18 \%$ in the Solitaire arm of SWIFT).

The low number of patients/year/center in the three trials probably indicates that all candidates for an IA treatment were not included in the trials and some of them may have been subjected to EVT outside the trial (compassionate use). Such an inclusion bias would severely affect the results of the trials, i.e., if patients likely to benefit from EVT had this treatment without being randomized.

Difficulty in the recruitment of patients was encountered in most RCTs dealing with endovascular treatment. This may, in part, explain the long-time periods needed to complete them. Mechanical thrombectomy is not yet a validated treatment and by not including all eligible patients in an RCT, physicians take the risk of skewing the results. Therefore, ongoing or future thrombectomy trials must address the requirements for centers enrolling patients. The same issue was previously encountered in the neurointerventional field with regard to carotid stenting trials

In conclusion, IMS III, SYNTHESIS, and MR RESCUE demonstrate that endovascular treatment is not appropriate for all patients with AIS. In addition, EVT is a heterogeneous group of very different techniques that do not have the same efficacy and should not be lumped together. One very consistent and highly important finding from the IMS III, SYNTHESIS, and MR RESCUE trials is that endovascular treatment is as safe as IV rTPA, and there are no safety issues that should deter the evaluation of the thrombectomy approach in more refined trials.

Evidence from trials evaluating the second-generation mechanical thrombectomy devices shows their superiority to the first-generation devices, indicating that the technique and the devices are evolving and becoming more effective. Future trials must focus on determining which treatments are the most efficacious and which patients will benefit from a particular treatment paradigm. Participation of both patients and physicians in randomized clinical trials is essential to provide rapid answers to this very important clinical problem.

These studies clearly shows that the selection of patients (clinical status evaluated with NIHSS, initial extension of ischemic lesions evaluated with ASPECT score, arterial occlusion depicted by CTA or MRA, evaluation of the salvageable brain evaluated by methods that have still to be precisely evaluated) and the therapeutic methods evaluated are key elements that must be addressed in the design of future studies. Patients with very low or high NIHSS have to be excluded as well as patients without arterial occlusion detected with CTA or MRA. It would be probably more productive to design studies to evaluate a single endovascular approach rather than evaluating a wide spectrum of endovascular approaches in the same study (e.g., IA chemical thrombolysis, mechanical thrombectomy performed in different ways or with very different generation devices). Also, comparison of the endovascular treatment alone to that of IV rTPA alone (as in SYNTHESIS and MR RESCUE) demands a highly effective local organization, in order to not lose time before EVT can be initiated. The combined approach (endovascular treatment + IV rTPA) allows the physician to start treatment early and to synergize the efficacy of chemical and mechanical thrombolysis (if there is a synergy).

Selection of the participating centers in the future RCTs in terms of physician competency and organization of the centers is certainly important to reduce the delays in the performance of EVT.

Finally, it is crucial that interventional neuroradiologists dealing with stroke participate to well-designed trials with new thrombectomy devices and that, in each participating center, all patients meeting the inclusion criteria are included in the trial (consecutive enrolment). If a pre-selection is applied 
by the center before randomization, the risk is that certain patients eligible for IA treatment are not randomized for "compassionate" reasons. This would create a serious inclusion bias and affect the results of the trial. As endovascular treatment has yet not proven its superiority to IV rTPA in a RCT, physicians cannot argue for treating patients outside of the trial.

Conflict of interest LP consults for Codman, Covidien/EV3, Microvention, Penumbra and Sequent. MS consults for Rapid Medical, Neuravi, Covidien/ev3 and has a research agreement with Philips Healthcare. MB consults for Codman. PW has research activity funded from Acandis, Codman, Covidien/ev3 and Microvention, consults for Microvention and has speaker bureau activities for Codman and Covidien/ev3. JG consults for Covidien/ev3. JF consults for Codman and Stryker and has speaker bureau activities for Covidien/ev3. CC consults for Codman, Covidien/ev3, Microvention, Sequent, and Stryker.

\section{References}

1. Broderick JP, Palesch YY, Demchuk AM et al (2013) Endovascular therapy after intravenous t-PA versus t-PA alone for stroke. N Engl J Med 368(10):893-903

2. Ciccone A, Valvassori L, Nichelatti M et al (2013) Endovascular treatment for acute ischemic stroke. N Engl J Med 368:904-913

3. Kidwell CS, Jahan R, Gornbein J et al (2013) A trial of imaging selection and endovascular treatment for ischemic stroke. N Engl $\mathrm{J}$ Med 368(10):914-923

4. Fiehler J, Söderman M, Turjman F et al (2012) Future trials of endovascular mechanical recanalisation therapy in acute ischemic stroke patients: a position paper endorsed by ESMINT and ESNR : part I: current situation and major research questions. Neuroradiology 54:1293-1301

5. Fiehler J, Söderman M, Turjman F et al (2012) Future trials of endovascular mechanical recanalisation therapy in acute ischemic stroke patients: a position paper endorsed by ESMINT and ESNR : part II: methodology of the future trials. Neuroradiology 54:1293-1301

6. Sutherland BA, Minnerup J, Balami JS et al (2012) Neuroprotection for ischaemic stroke: translation from the bench to the bedside. Int $\mathrm{J}$ Stroke 7:407-418

7. Hacke W, Kaste M, Fieschi C et al (1995) Intravenous thrombolysis with recombinant tissue plasminogen activator for acute hemispheric stroke: the European Cooperative Stroke Study (ECASS). JAMA 274:1017-1025

8. Multicenter Acute Stroke Trial -Italy (MAST-I) Group (1995) Randomised controlled trial of streptokinase, aspirin, and combination of both in treatment of acute ischaemic stroke. Lancet 346:1509-1514

9. The National Institute of Neurological Disorders and Stroke rTPA Stroke Study Group (1995) Tissue plasminogen activator for acute ischemic stroke. N Engl J Med 333:1581-1587

10. The Multicenter Acute Stroke Trial - Europ Study Group (1996) Thrombolytic therapy with streptokinase in acute ischemic stroke. N Eng J Med 335:145-150

11. Hacke W, Kaste M, Fieschi C et al (1998) Randomised double-blind placebo-controlled trial of thrombolytic therapy with intravenous alteplase in acute ischemic stroke (ECASS II). Lancet 352:12451251

12. Clark WM, Albers GW, Madden KP et al (2000) The rtPA (alteplase) 0- to 6-hour acute stroke trial, Part A (A0276g): results of a doubleblind, placebo-controlled, multicenter study. Stroke 31:811-816

13. Clark WM, Wissman S, Albers GW et al (1999) Recombinant tissuetype plasminogen activator (alteplase) for ischemic stroke 3 to 5 hours after symptom onset: the ATLANTIS study - a randomized controlled trial. JAMA 282:2019-2026

14. Hacke W, Kaste M, Bluhmki E et al (2008) Thrombolysis with alteplase 3 to 4.5 hours after acute ischemic stroke. N Engl J Med 359:1317-1329

15. Wahlgreen N, Ahmed N, Davalos A et al (2008) Thrombolysis with alteplase 3-4.5 h after ischaemic stroke (SITS-ISTR) an observational study. Lancet 372:1303-1309

16. Del Zoppo GJ, Higashida RT, Furlan AJ et al (1998) PROACT: a phase II randomized trial of recombinant pro-urokinase by direct arterial delivery in acute middle cerebral artery stroke. Stroke 29:4 11

17. Furlan A, Higashida R, Wechsler L et al (1999) Intraarterial prourokinase for acute ischemic stroke. The PROACT II study: a randomized controlled trial. JAMA 282:2003-2011

18. The IMS study investigators (2004) Combined intravenous and intraarterial recanalization for acute ischemic stroke: the Interventional Management of Stroke Study. Stroke 35:904-912

19. The IMS II trial investigators (2007) The Interventional Management of Stroke (IMS) II trial. Stroke 38:2127-2135

20. Ogawa A, Mori E, Minematsu K et al (2007) Randomized trial of intraarterial infusion of urokinase within 6 hours of middle cerebral artery stroke. The middle cerebral artery embolism local fibrinolytic intervention trial (MELT) Japan. Stroke 38:2633-2639

21. Smith WS, Sung G, Starkman S et al (2005) Safety and efficacy of mechanical embolectomy in acute ischemic stroke: results of the MERCI trial. Stroke 36:1432-1438

22. Smith WS, Sung G, Saver J et al (2008) Mechanical thrombectomy for acute ischemic stroke: final results of the Multi MERCI trial. Stroke 39:1205-1212

23. Penumbra pivotal stroke trial investigators (2009) The Penumbra pivotal stroke trial: safety and effectiveness of a new generation of mechanical devices for clot removal in intracranial large vessel occlusive disease. Stroke 40:2761-2768

24. Castano C, Dorado L, Guerrero C et al (2010) Mechanical thrombectomy with the Solitaire AB device in large artery occlusions of the anterior circulation. A pilot study. Stroke 41:1836-1840

25. Costalat V, Machi P, Lobotesis K et al (2011) Rescue, combined, and stand-alone thrombectomy in the management of large vessel occlusion stroke using the Solitaire device: a prospective 50-patient single -center study. Stroke 42:1929-1935

26. Soize S, Kadziolka K, Estrade L et al (2013) Mechanical thrombectomy in acute stroke: prospective pilot trial of the Solitaire FR Device while under conscious sedation. AJNR Am J Neuroradiol 34:360-365

27. Saver JL, Jahan R, Levy EI et al (2012) Solitaire flow restoration device versus Merci Retriever in patients with acute ischaemic stroke (SWIFT): a randomized, parallel-group, non-inferiority trial. Lancet 380:1241-1249

28. Nogueira RG, Lutsep HL, Gupta R et al (2012) Trevo versus Merci retrievers for thrombectomy revascularization of large vessel occlusions in acute ischaemic stroke (TREVO 2): a randomized trial. Lancet 380:1231-1240 